\title{
TEM STUDIES OF CONi/Pt MULTILAYERS
}

J.Rose, J.N.Chapman, Department of Physics and Astronomy, University of Glasgow, Glasgow G12 8QQ, UK J.C.Lodder, Q.Meng, MESA Research Institute, Universiteit Twente, P.O.Box 217, 7500 AE Enschede, The Netherlands

abstract - Transmission electron microscopy has been used to study the micromagnetic reversal mechanism in CoNi/Pt multilayers. Magnetisation reversal proceeds by nucleation at a small number of sites followed by domain wall branching to form an irregular dendritic domain structure. A substantial increase in field strength is required to eliminate the domains. Annealing at $300^{\circ} \mathrm{C}$ in vacuum leads to increased coercivity and a change in time dependent effects during the initial part of the reversal process .

KEYWORDS - CONi/Pt MULTILAYER, MAGNETISATION REVERSAL, LORENTZ MICROSCOPY.

\section{INTRODUCTION}

Extensive investigations have been made of $\mathrm{Co} / \mathrm{Pt}$ multilayers for magneto-optic recording ${ }^{[1,2]}$. Although such multilayers display many attractive properties, one problem encountered is the relatively high Curie temperature and, recently, $\mathrm{Ni}$ has been added to the magnetic layer in an attempt to reduce it $^{[3]}$. In this paper we describe the magnetisation processes in $\mathrm{Co}_{50} \mathrm{Ni}_{50} / \mathrm{Pt}$ multilayers from a microscopic stand point.

\section{Multilayer growth and processing}

The samples were prepared by sputter deposition. Initially the sputtering chamber was evacuated to a pressure of $5 \times 10^{-8} \mathrm{mbar}$, and then argon gas was admitted to a pressure of $1.6 \times 10^{-2}$ mbar. A typical film comprised a $27.5 \mathrm{~nm}$ Pt seed layer followed by 17 bilayers of $0.9 \mathrm{~nm} \mathrm{Co}_{50} \mathrm{Ni}_{50}+1.1 \mathrm{~nm}$ Pt. The deposition sequence was computer controlled. Following deposition, some of the samples were annealed in vacuum at $300 \mathrm{C}$. Films were deposited on electron transparent silicon nitride membranes supported on silicon wafers with windows etched into them. Samples of this kind were suitable for both the macroscopic and microscopic studies of their magnetic properties described below and all measurements were made on the same individual specimens.

\section{Experimental techniques}

Hysteresis loops were measured using a vibrating sample magnetometer (VSM). The sweep rate was 80 Oe. $s^{-1}$.

Studies of the domain processes that were involved as the samples were taken through their magnetisation cycles were made using a highly modified Philips CM20 transmission electron microscope (TEM). In addition to the standard objective lens the instrument is equipped with additional Lorentz (non-immersion) lenses which are used instead of the standard lens if domain structures are to be imaged without the specimen being subjected to a magnetic field ${ }^{[4]}$. Alternatively, images can be formed with the standard lens excited so that the vertical field at the specimen position lies anywhere in the range $-6.5 \mathrm{kOe}$ to $+6.5 \mathrm{kOe}$ thus facilitating studies of domain nucleation, propagation and annihilation. Hence domain observations can be made over a complete magnetisation cycle. The availability of the additional lenses provides greater flexibility than was the case in previous Lorentz microscopy studies ${ }^{[5]}$.

In the work reported here we primarily used the Fresnel (or defocus) imaging mode although other modes can be implemented where appropriate. Images were recorded digitally with exposure times of $0.25 \mathrm{~s}$ using a slow-scan CCD camera. As the multilayers under investigation support perpendicular magnetisation, it was necessary to tilt the samples to ensure there was a component of induction perpendicular to the electron beam, a necessity for all modes of Lorentz microscopy ${ }^{[6]}$.

\section{THE MAGNETISATION REVERSAL MECHANISM}

Figures 1(a) and (b) show the magnetisation loops from the specimens before and after annealing. In both cases the samples display $100 \%$ remanence. Reversal proceeds with a sharp fall in the magnetisation to zero followed by a more gradual increase to saturation in the reverse direction. The principal effect of annealing the sample is an increased coercivity, 6500 compared to 5100 e.

In a further VSM experiment, both samples were subjected to a saturating field in the positive direction followed by a negative field whose magnitude lay between the nucleation and saturation fields. On removal of the field, a substantial reduction in the 

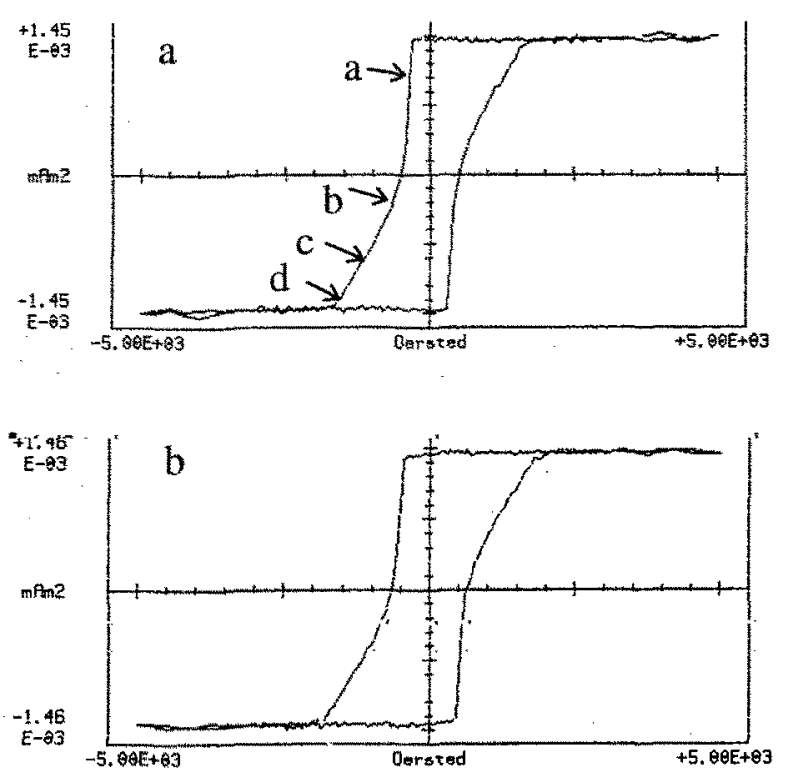

Figure 1: VSM loops (a) before annealing and (b) after annealing. The arrows in (a) show approximate positions in the loop where the images in figure 2 were recorded. magnetisation of the sample was observed, showing that reversible processes were not negligible. Hence a micromagnetic understanding of the form of the hysteresis loop requires that TEM observations are made with a field applied, rather than simply of remanent states. This was not the case in earlier studies of $\mathrm{Co} / \mathrm{Pt}$ multilayers which displayed considerably higher coercivities ${ }^{[7]}$.

Figure 2 shows domain structures observed during the magnetisation reversal process in an asgrown sample. The reversal process began from a very small number of sites and involved the growth of irregular, elongated domains (figure 2(a)). As the field was increased slightly the irregular domain structure grew to cover a greater fraction of the specimen (figure 2(b)). Observation of the process in real time, using a low-light-level TV camera coupled to a transmission phosphor, showed that growth involved multiple branching of existing domains to form the complex dendritic structures seen in the figure. Such structures have been observed previously in amorphous rare-
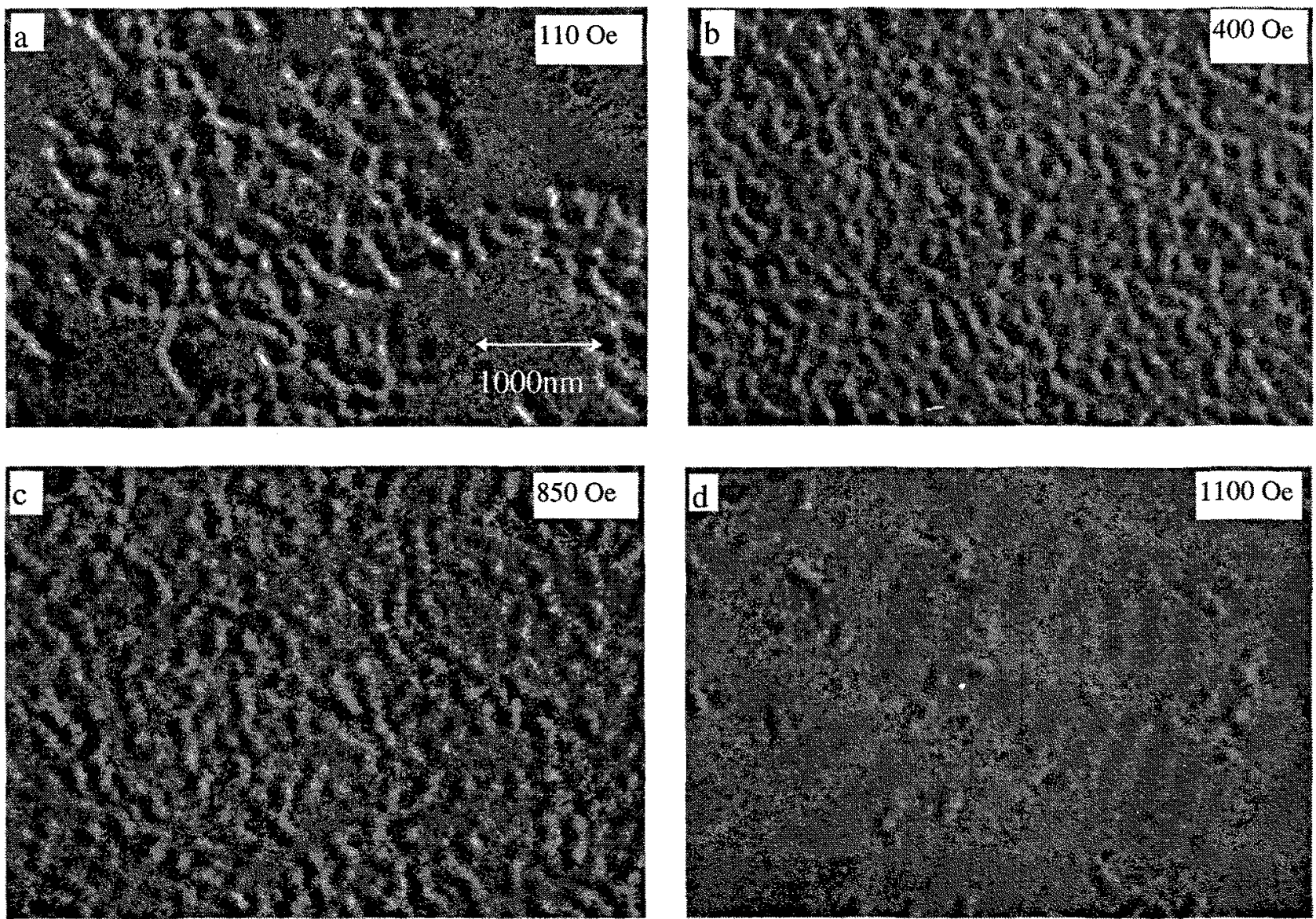

Figure2: The domain structure in the as grown sample at various points in the magnetic reversal field sequence. The fields are shown in the upper right hand corner of each image. 
earth/transition-metal (a-RETM) MO media ${ }^{[5,8]}$ and in $\mathrm{Co} / \mathrm{Pt}$ multilayers ${ }^{[7]}$. A further increase in field of $<100 \mathrm{Oe}$ led to the complete coverage of the sample with tightly-packed irregular domains (figure 2(b)), and once coverage was complete further changes required the application of substantially larger fields (figure 2(c)). Complete coverage appeared to coincide with a significant change in the gradient of the magnetisation curve close to where the total magnetisation is zero (figure 1). However, the existence of very significant time-dependent effects, discussed in the following section, made accurate confirmation of this point difficult.

The images clearly show that domain widths are on a sub-micron scale. From the Fresnel images shown, and from additional Foucault images, widths were estimated to lie typically in the range 180 to $250 \mathrm{~nm}$.

The remainder of the magnetisation process took place over a substantially larger field range and involved the steady shrinking of unfavourably oriented domains with the appearance of increased areas of reversed magnetisation separated by local regions supporting narrow irregular domains. Time-dependent effects were much less apparent. As the reverse field was increased, the number of regions supporting a domain structure decreased (figures $2(\mathrm{c})$ and (d)) until reverse saturation was attained. That extremely narrow domains existed up to substantial fields suggested that annihilation was difficult and may reflect the existence of wall structures of the kind predicted previously for multilayers supporting perpendicular magnetisation ${ }^{[9,10]}$.

The nucleation and subsequent growth of domains in the annealed sample closely resembled those in the as-grown state.

\section{TIME DEPENDENT PHENOMENA}

In the experiments described in this section, the specimen was subjected to a field slightly below the nucleation field as measured in the previous experiment and the nucleation and evolution of domains studied as
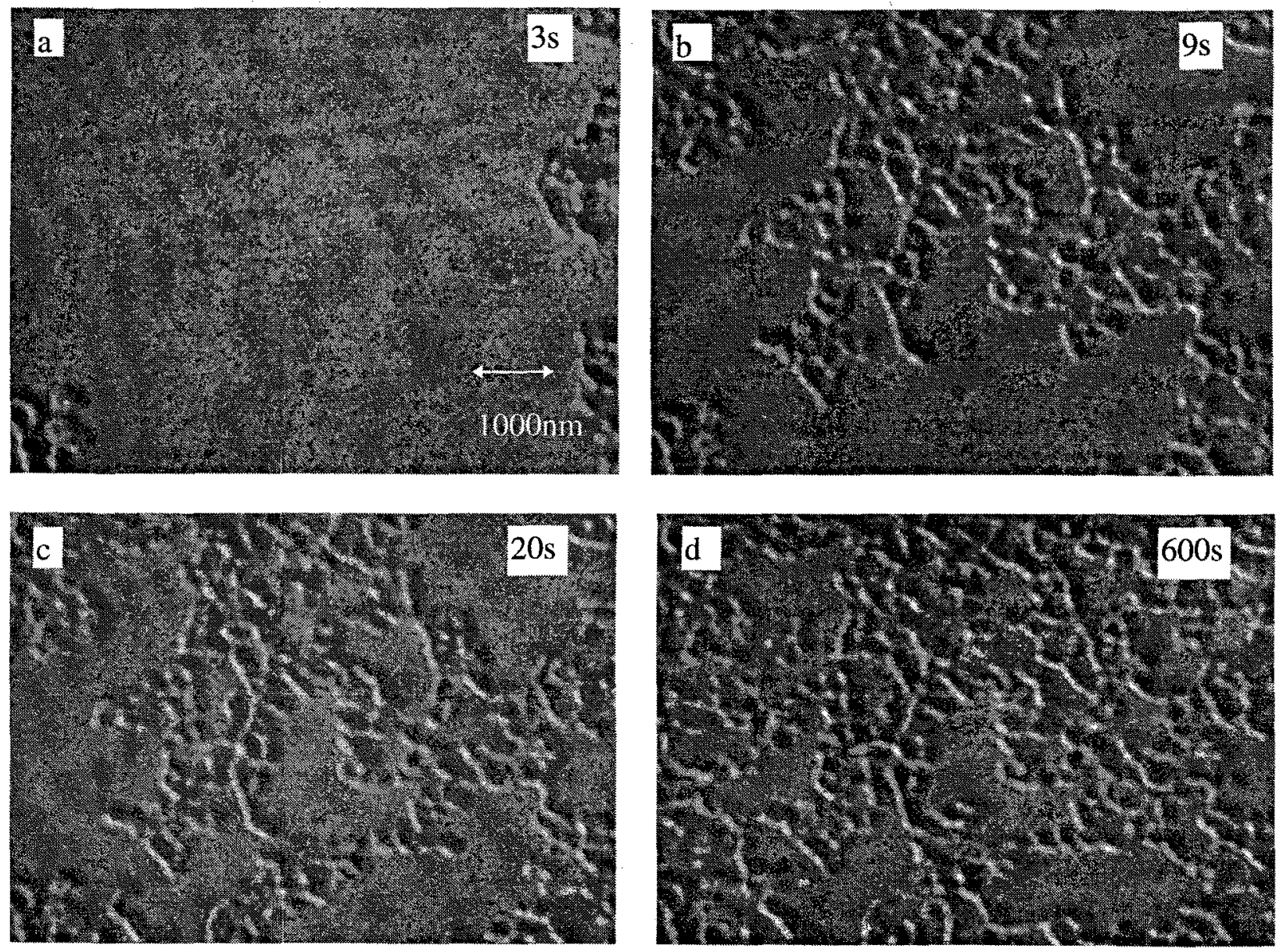

Figure 3: The change of the domain structure in the as grown sample with time. The images were recorded after the times shown in the upper right hand corner of each image. 
a function of time. Complete sequences were recorded directly on video tape using the low-light-level TV camera referred to above. Alternatively, lower noise images were recorded at regular intervals throughout the process by the following technique. After a preset time had elapsed the specimen tilt angle was modestly increased thereby reducing the vertical component of field at the specimen and hence effectively "freezing" the domain structure so that an image could be recorded using the $\mathrm{CCD}$ camera. Returning the specimen to its original tilt restored the field to its initial value and evolution of the domain structure continued. Figure 3 shows images taken in such a manner from an as-grown specimen; the times associated with each image are those for which the smaller tilt/higher field value applied. Sequences such as these show in greater detail how the dendritic domain structure spreads in an irregular manner across the specimen and confirms the importance of thermally activated effects in the initial part of the magnetisation cycle. Currently image analysis is in progress to determine how domain coverage increases as a function of both field and time and the magnitude of individual micro-Barkhausen jumps that can be distinguished within the time resolution of $20 \mathrm{~ms}$ attainable when the TV camera is used.

Whilst results from the annealed specimen are broadly similar, a difference does exist in that, following initial nucleation, the spread of domains to cover the specimen is more complete.

\section{DISCUSSION AND CONCLUSIONS}

The results presented here show how a TEM modified for enhanced performance in the study of magnetic materials can be used to provide a detailed picture of the magnetisation processes in multilayers of interest for MO recording. The phenomena observed in the $\mathrm{CoNi} / \mathrm{Pt}$ multilayers are qualitatively similar to processes seen previously in some a-RETM media and in $\mathrm{Co} / \mathrm{Pt}$ multilayers. Following nucleation, the development of an irregular dendritic domain structure suggests that the demagnetising energy is relatively. large, the wall energy is fairly low and the growth of reverse magnetisation is dominated by the geometry of the pinning sites ${ }^{[12]}$. Strongly time-dependent effects attest to the importance of thermally activated processes during the initial part of the magnetisation cycle. The differences observed between the annealed and as-grown samples may be due to an increase in nucleation coercivity following heat treatment whilst the wall-motion coercivity is less affected. That changes proceed more slowly once domains cover the entire specimen is not surprising in that the demagnetising effects which assisted the applied field in the initial part of the cycle now oppose it with the results that substantial field changes are required to shrink and subequently annihilate unfavourably oriented domains. Future work will focus on analysing image sequences to try to extract quantitative data on energy barriers and activation volumes associated with nucleation and wall-pinning in these and related films of interest for MO recording. Such information is necessary if irregularity in written marks, a factor believed to influence the CNR ratio, is to be understood.

\section{ACKNOWLEDGEMENTS}

The support of CAMST, the UK EPSRC and the EC HCM programme (CHRX-CT93-0316) is gratefully acknowledged for various aspects of this work.

\section{REFERENCES}

[1] W.B.Zeper, H.W.van Kesteren, B.A.J.Jacobs and J.H.M.Spruit, J. Appl. Phys. 70, 2264 (1991).

[2] T.Suzuki, H.Notarys, D.C.Dobbertin, C.J.Lin, D.Weller; D.C.Miller, G Gorman, IEEE Trans Mag. 28, 2754 (1992).

[3] Q.Meng, W.P.van Drent, J.C.Lodder and Th.J.A.Popma, Proceedings of MML'95, Cambridge, (1995)

[4] J.N.Chapman, A.B.Johnston, L.J.Heyderman, S.McVitie, W.A.P.Nicholson and B.Bormans, IEEE Trans. Magn, 30, 4479 (1994).

[5] C-J.Lin, J.C.Sui, and R.H.Geiss, J.Appl. Phys. 63, 3835 (1988).

[6] J.N.Chapman, J.Phys. D:Appl. Phys. 17, 623 (1984).

[7] D.M.Donnet, V.G.Lewis, J.N.Chapman, K.O'Grady, H.W.van Kesteren, J.Phys D:Appl Phys 26, 1741 (1993).

[8] M.Labrune, S.Andrien, F.Rio, P.Bernstein J.Magn. Magn.Mat. 80, 211 (1981).

[9] R.Ploessl, J.N.Chapman, M.R.Scheinfein, J.L.Blue. M.Mansuripur, H.Hoffmann, J.Appl.Phys. 74, 7431 (1993).

[10] T.Schrefl, J.Fidler and J.N.Chapman, submitted to J.Phys. D: Appl. Phys.

[11] J.N.Chapman, L.J.Heyderman, S.McVitie and W.A.P.Nicholson, Proc. 2nd NIRIM Int. Symp. on Advanced Materials (ISAM '95) 123 (1995).

[12] A.Lyberatos, J.Earl and R.W.Chantrell, submitted to Phys. Rev. B. 